\title{
Protective Effects of Small Peptides from Periplaneta americana on Cyclophosphamide-induced Oxidative Stress in Rat Ovaries
}

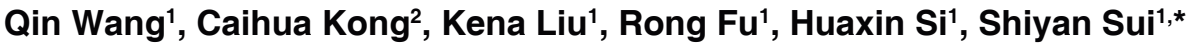 \\ ${ }^{1}$ School of Public Health, Dali University, Dali, Yunnan, CHINA. \\ ${ }^{2}$ College of Agronomy and Biological Sciences, Dali University, Dali, Yunnan, CHINA.
}

\begin{abstract}
Introduction: Periplaneta americana ( $P$. americana), commonly known as the American cockroach, has shown multiple clinical benefits. Small Peptides from $P$. americana (SPPA) was found to have promising antioxidative effects in vitro. However, the pharmacological benefits of SPPA demand further in-depth investigation as the underlying mechanism for its antioxidative effect remain unexplored. Objectives: To evaluate the effects of SPPA on ovarian follicles and antioxidant capacity in rats. Materials and Methods: In vivo model of oxidative stress, i.e., Sprague-Dawley (SD) rats with cyclophosphamide (CP)induced oxidative stress, was used to evaluate the effects of SPPA. Results: CP induced a significant decrease in the ovary weight and ovary/body weight ratio in both high- and/ or low- SPPA dose groups of rats. This effect was accompanied by down-regulated levels of MDA and NO and elevated levels of SOD and GSH-Px in the serum. Besides, both SOD protein and SOD mRNA expression levels in the ovary were elevated. Rats treated with a higher SPPA dose showed a higher number of Graafian follicles and lower atretic follicles and Ki67 mRNA was upregulated and both caspase-3 and Fasl mRNA expression levels were down-regulated. Conversely, the $\mathrm{CP}$ group of rats showed an incessant decrease in the number of normal follicles and an increase in the number of atretic follicles, along with the enhanced expression of BMP4, GDF9 and IGF1 mRNAs. Conclusion: This study indicates that SPPA acts as potent antioxidant and might be developed as a promising therapeutic agent for inhibiting ovarian injury.
\end{abstract}

Key words: Small Peptides from Periplaneta americana, Oxidative stress, Gene expression, Ovarian follicle, Cyclophosphamide, Rats.

\section{INTRODUCTION}

The disparity between the reactive oxygen species (ROS) generation and abrogation gives rise to cellular oxidative stress. Excessive oxidative stress damages tissues and disrupts cellular redox circuits. ROS participates in multiple biological processes, such as oogenesis, oocyte maturation and follicular formation. However, excessive ROS reacts with cellular lipids, culminating in lipid peroxidation. The toxic and aldehyde moieties containing by-products of lipid peroxidation, such as Malondialdehyde (MDA) and nitric oxide (NO), indicate cell damage due to lipid peroxidation and ovarian dysfunction. ${ }^{1,2}$ Incessant and elevated oxidative stress disrupts the structure and function of cellular DNA, culminating in cell dysfunction and even cell death. ${ }^{3}$ Furthermore, oxidative stress results in a myriad of ovarian disorders, such as endocrine disorders, premature ovarian failure, follicular atresia and so on. Besides, it also leads to embryo aging, stunted development of the fetus and numerous adverse effects in human-assisted reproduction. ${ }^{1}$ Thus, balancing oxidative stress in the ovary could improve the oocyte quality and stimulate embryo proliferation.

Multiple studies have shown that follicles contain glutathione peroxidase (GSH-Px),
Submission Date: 23-01-2021; Revision Date: 01-04-2021; Accepted Date: 27-05-2021

DOI: 10.5530/ijper.55.3.151 Correspondence:

Dr. Shiyan Sui

School of Public Health, Dali University, Dali,

Yunnan-671000, CHINA. Phone: +86-872-2257380 Email id: sysui569@163.com

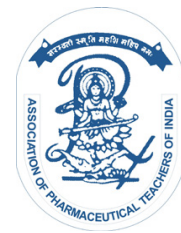

www.ijper.org 
catalase (CAT) and superoxide dismutase (SOD) as endogenous and primary antioxidant enzymes in eukaryotic cells. ${ }^{3}$ SOD catalyzes $\mathrm{O}_{2}$ - to generate $\mathrm{H}_{2} \mathrm{O}_{2}$, which is subsequently decomposed to water and oxygen by CAT. GSH-Px catalyzes the conversion of GSH to glutathione disulfide (GSSG), which in conjugation with NADPH, reduces the toxic effects of free radicals. GSH-Px depletes the ROS content in follicles and impedes oxidative stress along with non-enzymatic antioxidants. ${ }^{2}$ To neutralize the ROS toxicity, exogenous free radical scavengers block the free radicals, preventing the harmful effects of oxidative damage. ${ }^{1-3}$

P. americana is mentioned as "Shen Nong Ben Cao Jing" in the ancient Chinese pharmacopeia and is commonly known as the American cockroach; it is native to South America and occurs worldwide. It mostly feeds on sugar and starch and mostly involved in polluting the food and spreading infection. Although secretions and feces of $P$. americana contain carcinogens, $P$. americana has shown multiple pharmacological benefits in humans. ${ }^{4}$ It shows anti-tumor, anti-hepatitis, anti-bacterial, antihuman immunodeficiency, antioxidant and analgesic effects $^{5}$ and promotes vascular proliferation, tissue repair, immunity and suppresses inflammation. ${ }^{5}$ The fresh product of Periplaneta americana was extracted by alcohol-water extraction, concentration, degreasing, macroporous adsorption resin column chromatography, alcohol solvent elution and other procedures to obtain a peptide-based refined extract (i.e SPPA), which was identified Small peptides in this substance account for $94.44 \%$ (Table 1 and Figure 1). SPPA was found to have promising antioxidative effects in vitro and has since become increasingly popular as a therapeutic agent in China. ${ }^{4}$ However, it demands further in-depth investigation as the underlying mechanism for the antioxidative effect of SPPA remains unexplored.

\begin{tabular}{|c|c|c|c|}
\hline \multicolumn{5}{|c|}{ Table 1: The molecular weight range and peak area } \\
ratio of SPPA. \\
$\begin{array}{c}\text { Serial } \\
\text { number }\end{array}$ & $\begin{array}{c}\text { Retention } \\
\text { time(min) }\end{array}$ & $\begin{array}{c}\text { The range } \\
\text { of molecular } \\
\text { weight (Da) }\end{array}$ & $\begin{array}{c}\text { Percentages of } \\
\text { the total peak } \\
\text { area (\%) }\end{array}$ \\
\hline 1 & $16.47-34.18$ & $12355-612.6$ & 62.17 \\
\hline 2 & $34.18-38.72$ & $612.6-268$ & 22.24 \\
\hline 3 & $38.72-40.51$ & $268-181$ & 12.02 \\
\hline 4 & $\geq 2.028$ & - & 3.58 \\
\hline
\end{tabular}

Draw the standard curve with the peak time (tR/min) of the standard substance (bovine serum albumin) as the $\mathrm{X}$-axis and the logarithmic value Log10 (Mr) of the molecular weight as the $\mathrm{Y}$-axis and then obtain the regression equation and put the sample peak time into the curve equation to calculate each the relative molecular mass of the peak is calculated by the peak area normalization method to calculate the relative percentage of substances in different molecular weight ranges. The results come from the research group of $P$. americana at Dali University.
Growth differentiation factor-9 (GDF-9) plays a crucial role in the development of primordial follicles during folliculogenesis. ${ }^{6}$ Bone morphogenetic protein 4 (BMP4) ameliorates the transformation of primordial follicles to primary follicles. ${ }^{7}$ Additionally, an increased level of insulin-like growth factors (IGF-1) is essential for follicular maturation and development. ${ }^{8}$ Cellular apoptosis and follicular atresia are common physiological phenomena associated with follicular development. However, aberrant apoptosis can reduce the number of available follicles and inhibit the reproductive efficiency of women. Three primary mechanisms for cellular apoptosis are a) Cell surface receptor pathway (apoptosis protein-1/Fas ligand pathway), ${ }^{1}$ b) mitochondria-mediated apoptosis pathway [B-cell lymphoma-2 (Bcl-2) family activation, the ratio of $\mathrm{BAX} / \mathrm{Bcl}-2$ is often used to evaluate the apoptosis level of follicles], ${ }^{2}$ c) endoplasmic reticulum stressmediated death pathway. Caspase-3 is a commonly used apoptosis indicator. ${ }^{10}$ The ki-67 antigen is a commonly used indicator of follicular development. ${ }^{11}$

Cyclophosphamide (CP) is a widely used alkylating anticancer drugs. CP is closely associated with ovarian disorders. As shown in previous studies, a higher dose of CP caused irreversible damage to oocytes. ${ }^{12}$ Also, prolonged treatment with CP reduced oocyte counts, induced early menopause and promoted ovarian failure. ${ }^{13}$ Thus, this study aimed to evaluate the effect of $P$. americana extract (SPPA) on the number of ovarian follicles along with changes associated with oxidative or antioxidative markers in the serum of adult Sprague-Dawley (SD) rats. Furthermore, the expression levels of genes related to

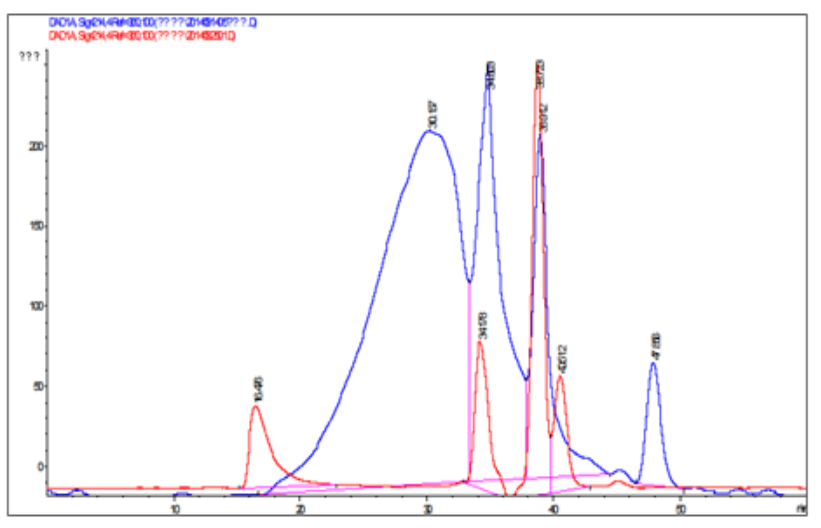

Figure 1: Overlay chromatogram of molecular weight distribution of reference substance and sample. The blue is the High Performance Liquid Chromatography (HPLC) chart of SPPA; the red is the HPLC chart of the 4 standard products. The standard products are from left to right: cytochrome $\mathbf{C}$, oxidized glutathione, inosine and tyrosine acid. The results come from the research group of $P$. americana at Dali University. 
antioxidant generation, ovarian development, apoptosis and proliferation in rat ovaries were investigated. The outcomes of this study will extend our understanding of the antioxidative effect of SPPA in rat ovaries. Also, it will provide platform data to reveal the impact of SPPA on reproductive functions.

\section{MATERIALS AND METHODS}

\section{Reagents}

Small Peptide from Periplaneta americana extract (SPPA) was provided by the professor of Guangming Liu in Dali university, China (no. 20200830). Cyclophosphamide (Baxter Company, Germany). NO, MDA, CAT, GSH-Px and SOD kits (Nanjing Jiancheng Bioengineering Institute, China). Paraformaldehyde (Chemical reagent Co., Ltd. of Shanghai Ling Feng, China). TRIzol kit (Biotechnology Company of Shanghai Life Technologies, China). The reverse transcription kit (Biochemical Technology Company of Beijing Tiangen, China). DNA polymerase containing SYBR Green (Bao Bioengineering Company of Dalian, China). Reverse transcription primers, internal reference primers and gene expression primers were synthesized by Genewiz Company, Suzhou.

\section{Small Peptides from Periplaneta americana}

Adult $P$. americana were air-dried and pulverized using an air dryer and a crusher, respectively and its extract was prepared as described previously. ${ }^{5}$ Briefly, a cold-soak extraction method was used where pulverized powder of $P$. americana was extracted using a 15 times volume of $95 \%$ ethanol thrice. The resulting precipitate was stored at $-40^{\circ} \mathrm{C}$ after removing the fat. Then, $P$. americana was extracted by macroporous adsorption resin column chromatography, alcohol solvent elution and other procedures to obtain a peptide-based refined extract (i.e SPPA), 181 12355 Da, which was identified Small peptides in this substance account for $94.44 \%$ (Table 1 and Figure 1). SPPA was diluted using distilled water. High-dose group contained $9 \mathrm{mg} / \mathrm{mL}$ and low-dose group contained $3 \mathrm{mg} / \mathrm{mL}$ of SPPA.

\section{Experimental animals}

Animal dissection and tissue extraction were in accordance with the "Guidelines on Ethical Treatment of Experimental Animals (2006) No. 398," Ministry of Health Science and Technology, the People's Republic of China. The project was approved by the Animal Ethics Committee at Dali University (Project number: 31760719).
A total of 32 female SD rats with an average weight of around 230-250 $\mathrm{g}$ were provided by the experimental animal center of Kunming Medical University. Following one week of acclimatization, rats were randomly divided into four groups $(n=8)$, according to the random number table and rat weight.

Rats that received an intraperitoneal injection of saline were treated as the control, whereas the rats that received $150 \mathrm{mg} / \mathrm{kg}$ of CP in a single dose for $5 \mathrm{~d}$ were treated as the positive control. Later these rats were gavaged with saline for consecutive 25 days. Concurrently, the rats in the high-dose ( $9 \mathrm{mg} / \mathrm{mL}$ SPPA) and low-dose ( $3 \mathrm{mg} / \mathrm{mL}$ SPPA) groups were administered $150 \mathrm{mg} / \mathrm{kg}$ of CP through intraperitoneal injection for the first $5 \mathrm{~d}$ and later gavaged with the corresponding dose of SPPA for consecutive 25 days. All four groups of rats received the same dose $(9 \mathrm{~mL} / \mathrm{kg})$ of saline or SPPA. ${ }^{4,5}$ The body weight was measured once every $3 \mathrm{~d}$.

\section{Tissue collection}

On the $31^{\text {st }}$ day of the experiment, rats were anesthetized with an intraperitoneal injection of $10 \%$ chloral hydrate $(2 \mathrm{~mL} / \mathrm{kg})$. Blood was collected during the dissection of the abdominal cavity and the plasma was separated from the blood. The right ovaries of rats were harvested immediately after dissection, stored at $-80^{\circ} \mathrm{C}$ and the left ovaries were fixed in $4 \%$ paraformaldehyde $(0.1 \% \mathrm{M}$, $\mathrm{pH}$ 7.2) solution.

\section{Classification of different grades of follicles}

The left ovaries were embedded in paraffin using the conventional methods as described previously. ${ }^{3,14} \mathrm{Six} \mu \mathrm{m}$ thick sections were cut from paraffin-embedded tissue using a digital pathology slice scanner and later stained using hematoxylin and eosin. Follicles were divided into primordial follicles, primary follicles, secondary follicles, antral follicles and Graafian follicles as per the morphological classification of follicles by Cheng $\mathrm{G}$ et al. ${ }^{15} \mathrm{~A}$ computer connected to a light microscope was used for digitizing the images and the first three types of follicles, i.e., primordial follicles, primary follicles and secondary follicles, were observed at 400x magnification and the latter two types of follicles, i.e., antral follicles and Graafian follicles were observed at 100x magnification.

\section{Determination of oxidative/antioxidative stress indexes in plasma}

The levels of NO, MDA, CAT, GSH-Px and SOD in plasma were determined using the respective kits (Nanjing Jiancheng Bioengineering Institute, China), as per the manufacturer's instruction. 


\section{Reverse transcription-quantitative polymerase chain reaction ( $q R T-P C R$ )}

Total RNA was extracted from the right ovaries of rats using the TRIzol reagent (Life Technologies, China) and subsequently treated with DNase I (Takara, Japan). The RNA concentration was determined using a spectrophotometer. After checking the RNA integrity and DNA contamination, $2 \mu \mathrm{g}$ of total RNA was reverse-transcribed using a reverse transcription kit (Tiangen, China), as per the manufacturer's instructions. For qRT-PCR, $2 \mu \mathrm{L}$ of $\mathrm{cDNA}$ was diluted 20 times. All primers (Table 2) were designed using target gene sequences from GenBank and synthesized by Genewiz Co., Ltd. (Suzhou, China). Glyceraldehyde-3-phosphate dehydrogenase (GAPDH) was used as a reference gene. The specificity of each primer was checked via meltingcurve analysis and PCR product sequencing.

\section{Protein extraction and western blotting}

Protein was extracted from frozen ovaries as described previously. ${ }^{16}$ Protein concentration was estimated using the BCA protein assay kit (Pierce, USA). $40 \mu \mathrm{g}$ of ovarian protein was loaded on $12 \%$ SDS-PAGE gel and resolved electrophoretically. For western blot analysis, primary antibodies used were SOD (Abcam, USA, 1: 1000) and $\beta$-actin antibody (internal reference) (Bioworld, China, 1: 10000).

\section{Statistical analysis}

All data were presented as mean \pm SEM. All data were analyzed using one-way ANOVA by employing SPSS version 21.0. Real-time PCR data were analyzed using $2^{-\Delta \Delta \mathrm{Ct}}$ method to evaluate relative expression levels of genes. The expression levels of gene and protein, the proportion of follicles were expressed as the fold

\begin{tabular}{|c|c|c|c|}
\hline Genes & $\begin{array}{c}\text { PCR } \\
\text { product (bp) }\end{array}$ & Sequence $\left(5^{\prime}-3^{\prime}\right)$ & GenBank ID \\
\hline CAT & 213 & $\begin{array}{l}\text { F: ACCTGTGAACTGTCCCTACCG } \\
\text { R: CGCACCTGAGTGACGTTGTCT }\end{array}$ & NM_012520 \\
\hline SOD & 163 & $\begin{array}{l}\text { F: GTGGTGGAGAACCCAAAGGA } \\
\text { R: GCGGCAATCTGTAAGCGAC }\end{array}$ & NM_017051 \\
\hline GSH-Px & 221 & $\begin{array}{l}\text { F: GTGCGAGGTGAATGGTGAGA } \\
\text { R: TGTCGATGGTGCGAAAGC }\end{array}$ & NM_030826 \\
\hline BMP4 & 188 & $\begin{array}{l}\text { F: CAGGGCCAACATGTCAGGAT } \\
\text { R: TGGCGACGGCAGTTCTTATT }\end{array}$ & NM_012827 \\
\hline GDF9 & 130 & $\begin{array}{l}\text { F: TGCCTGGCTGTGTCTTCTTATT } \\
\text { R: GTCAGTCCCATCTACAGGCAA }\end{array}$ & NM_021672 \\
\hline IGF1 & 162 & $\begin{array}{l}\text { F: GACCCGGGACGTACCAAAAT } \\
\text { R: GAACTGAAGAGCGTCCACCA }\end{array}$ & NM_178866 \\
\hline BAX & 152 & $\begin{array}{l}\text { F: GGCGATGAACTGGACAACAA } \\
\text { R: GCAAAGTAGAAAAGGGCAACC }\end{array}$ & NM_017059 \\
\hline $\mathrm{Bcl}-2$ & 101 & $\begin{array}{l}\text { F: TATAAGCTGTCACAGAGGGGCTAC } \\
\text { R: TCAGGCTGGAAGGAGAAGATG }\end{array}$ & NM_016993 \\
\hline Casp3 & 212 & $\begin{array}{l}\text { F: CCGATGTCGATGCAGCTAAC } \\
\text { R: TTTCAGGTCCACAGGTCCGT }\end{array}$ & NM_012922 \\
\hline FasL & 136 & $\begin{array}{l}\text { F: ACCACCTCCATCACCACTACC } \\
\text { R: CATTCCAACCAGAGCCACC }\end{array}$ & NM_001205243 \\
\hline Fas & 177 & $\begin{array}{l}\text { F: CTGTGATGAAGGGCATGGTTT } \\
\text { R: TTGGTGTTGCTGGTTCGTGT }\end{array}$ & NM_139194 \\
\hline Ki67 & 211 & $\begin{array}{l}\text { F: GACAGTGGAGTGGCTTTTGTGA } \\
\text { R: AAAGCCCCTTGGCATAGACA }\end{array}$ & NM_001271366 \\
\hline GAPDH & 142 & $\begin{array}{l}\text { F: GGCAAGTTCAACGGCACAG } \\
\text { R: CGCCAGTAGACTCCACGACAT }\end{array}$ & NM_017008 \\
\hline
\end{tabular}


change compared with control. $P<0.05$ was considered statistically significant.

\section{RESULTS}

\section{Changes associated with ovary and body weight of rats}

During the initial experimental stage, the average weight of rats in all four groups $(P>0.05)$ did not differ significantly. However, at the last stage of the experiment, the average body weight (BW), ovary weight (OW) of the rats and $\mathrm{OW} / \mathrm{BW}$ ratio in the high-dose group and control group of rats were significantly higher $(P<$ $0.05)$ and $\mathrm{OW} / \mathrm{BW}$ ratio in the low-dose group of rats was higher $(P=0.06)$ than $\mathrm{CP}$ group of rats. Concisely, $\mathrm{BW}, \mathrm{OW}$ and $\mathrm{OW} / \mathrm{BW}$ ratio did not differ significantly between the $(P>0.05)$ controls, high- and low-dose groups of rats (Table 3$)$.

\section{Quantification of follicles in the development stages}

Quantification of follicles at different developmental stages is depicted in Figure 2. A significant decrease in the number of primordial, primary, secondary, antral and Graafian follicles $(P<0.05)$ and a significant increase in the number of atretic follicles $(P<0.05)$ were observed in the CP group of rats as compared to the control group of rats. The high-dose group also demonstrated a significant increase in the number of Graafian follicles $(P<0.05)$. In contrast to the CP group, the number of atretic follicles in the high-dose group of rats decreased significantly $(P<0.05)$. Besides, the number of Graafian follicles in both the high- $(P<0.01)$ and low-dose $(P<$ $0.05)$ groups of rats increased significantly.
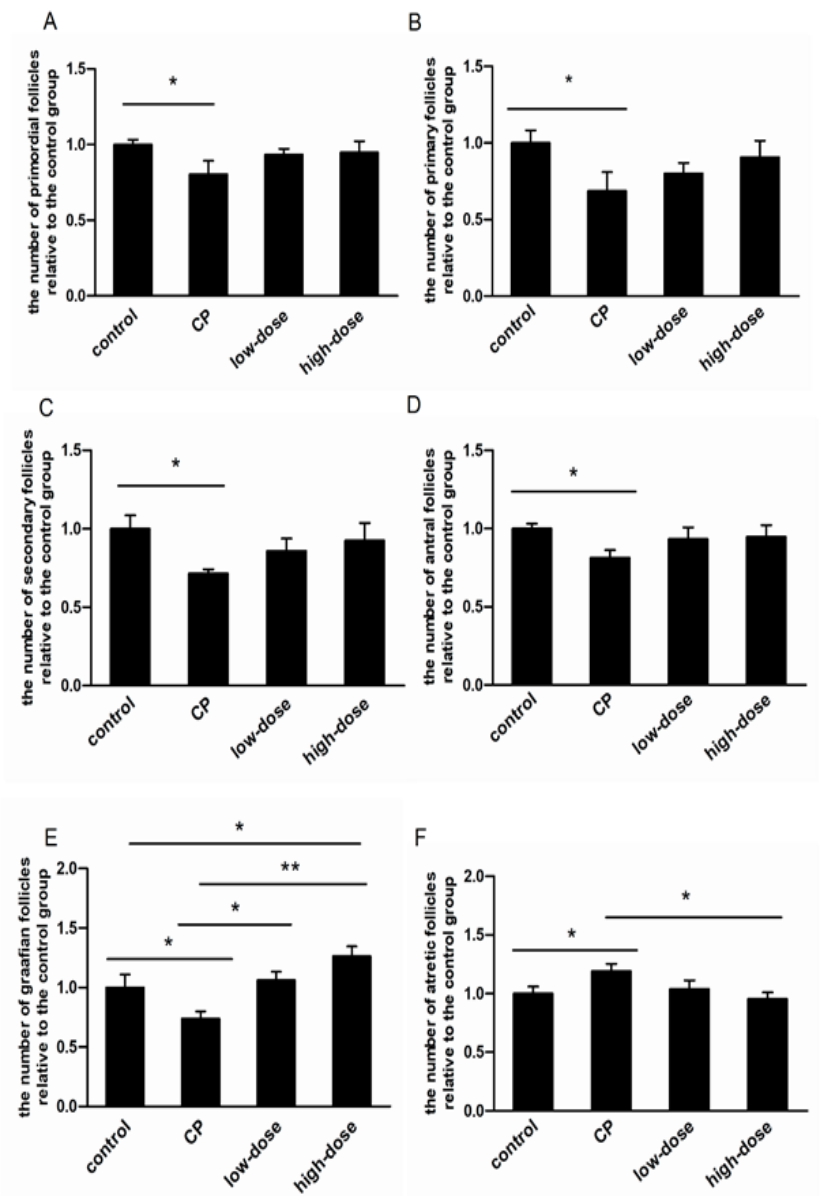

Figure 2: The number of different ovarian follicles in rats. A-F, primordial follicle; primary follicle; secondary follicle; antral follicle; Graafian follicle; atretic follicle. Control, the control group; CP, the cyclophosphamide (CP) group; low-dose, the low-dose SPPA and CP group; high-dose, the high-dose SPPA and CP group. Data was a fold-change in the control group. Three sections of each ovary were analyzed to quantitate the follicles. Values are shown as mean+SEM and each group had 8 rats. ${ }^{*}(P<0.05)$ and ${ }^{* *}(P<0.01)$ represent statistically significant differences.

\begin{tabular}{|} 
Table 3: Bodyweight, ovary weight and the ratio of ovary/body weight in \\
different groups of rats. \\
\hline Parameters & Control group & CP group & $\begin{array}{c}\text { Low-dose } \\
\text { group }\end{array}$ & $\begin{array}{c}\text { High-dose } \\
\text { group }\end{array}$ \\
\hline Initial BW (g) & $245.28 \pm 3.52$ & $246.45 \pm 3.26$ & $245.20 \pm 3.03$ & $246.42 \pm 3.18$ \\
Final BW (g) & $321.76 \pm 2.95^{\mathrm{a}}$ & $302.96 \pm 4.70^{\mathrm{b}}$ & $316.91 \pm 7.39 \mathrm{a}^{\mathrm{b}}$ & $319.26 \pm 4.42^{\mathrm{a}}$ \\
\hline OW (mg) & $80.87 \pm 4.04^{\mathrm{a}}$ & $60.49 \pm 6.22^{\mathrm{b}}$ & $73.18 \pm 4.22 \mathrm{a}^{\mathrm{b}}$ & $79.14 \pm 4.00^{\mathrm{a}}$ \\
\hline $\begin{array}{c}\text { OW/BW } \\
\text { (mg/100g) }\end{array}$ & $24.98 \pm 1.28^{\mathrm{a}}$ & $19.95 \pm 1.96^{\mathrm{b}}$ & $23.12 \pm 1.28^{\mathrm{ab}}$ & $24.76 \pm 1.12^{\mathrm{a}}$ \\
\hline
\end{tabular}

OW, ovary weight; BW, body weight; OW/BW, the ratio of the ovary to body weight. Values are shown mean $\pm \mathrm{SEM}$, $P<0.05$ indicates statistical significance. Lowercase letters ( $a$ and $b$ ) indicate the significant difference; if it contains the same letters on the shoulder between different data of peer data indicate no difference. 


\section{Effects of SPPA on oxidative/antioxidative stress markers in the rat's blood}

$\mathrm{NO}$ and MDA (Figure 3A and Figure 3B) levels were increased $(P<0.05)$ and GSH-Px and SOD levels were decreased significantly in the CP group of rats as compared to the control group of rats $(P<0.05)$ (Figure $3 \mathrm{D}$ and Figure $3 \mathrm{E})$. However, no significant differences were observed between these four indicators in the highand low-dose groups of rats. The high- and low-dose groups of rats demonstrated a significantly decreased MDA $(P<0.05)$ and increased SOD $(P<0.05)$ levels than the $\mathrm{CP}$ group of rats. In addition, the high-dose group showed a significantly reduced $(P<0.05) \mathrm{NO}$ level, similar to the low-dose group $(P=0.063)$ of rats. CAT levels did not differ significantly between the four groups of rats (Figure 3C).
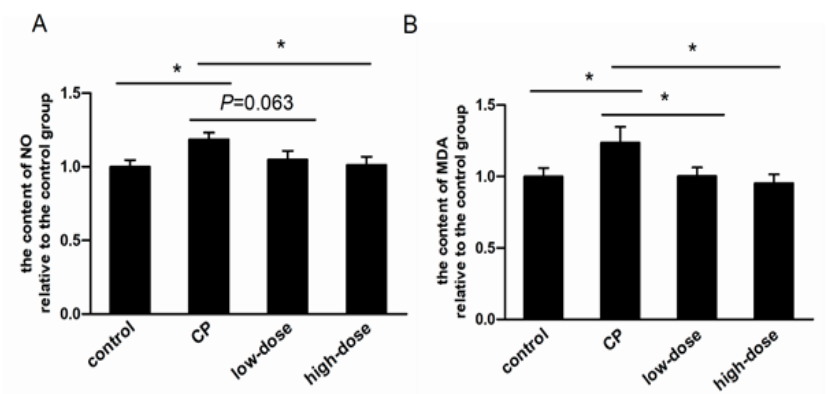

C

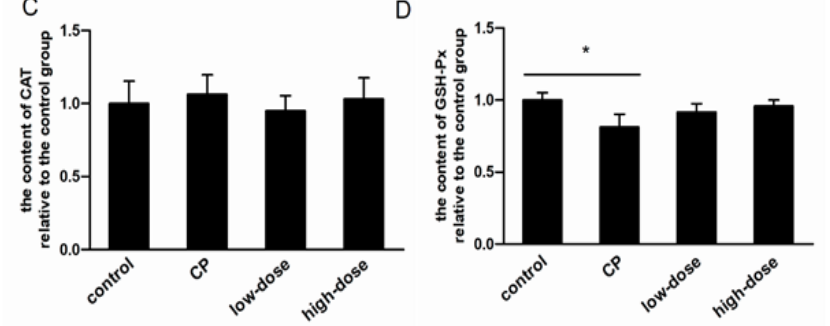

E

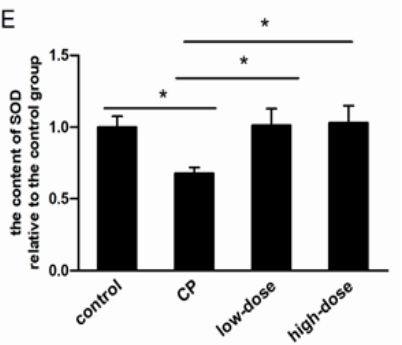

Figure 3: NO, MDA, CAT, GSH-Px and SOD levels in the blood of rats. A) NO; B) MDA; C) CAT; D) GSH-Px; E) SOD. Control, the control group; CP, the cyclophosphamide (CP) group; low-dose, the low-dose SPPA and CP group; high-dose, the high-dose SPPA and CP group. Data was fold-change of the control group. Values are shown as mean \pm SEM and each group had eight rats. * represent statistically significant differences $(P<0.05) . P=0.063$ indicates that the NO level in the blood of the low-dose group of rats tended to be lower than the CP group of rats.
Ovarian mRNA expression of antioxidant stressrelated biomarkers

The mRNA expression levels of CAT and GSH-Px did not differ significantly between the four groups of rats (Figure 4A and Figure 4B). SOD mRNA expression decreased significantly in the $(P<0.05) \mathrm{CP}$ group as compared to the control group of rats; however, it did not differ significantly in the high- and low-dose groups of rats. Moreover, SOD protein expression was also significantly lower $(P<0.05)$ in the CP group (Figure 4) of rats. The $S O D$ mRNA expression was significantly up-regulated $(P<0.05)$ in both high- and low-dose groups (Figure 4C) as compared to the CP group of rats. In addition, SOD protein level was significantly up-regulated in the high-dose group of rats $(P<0.05)$ (Figure 5).

\section{mRNA expression of ovarian development genes}

The BMP4, GDF9 and IGF1 mRNA expression levels in the $\mathrm{CP}$ group were significantly up-regulated $(P<0.05)$ and remained unaltered in the high- and low-dose groups as compared to the control group of rats. However, BMP4 and GDF9 mRNA expression was significantly reduced $(P<0.05)$ in high- and low-dose groups of rats as compared to CP group of rats (Figure 6).

mRNA expression of ovarian apoptosis and proliferation-related genes

No significant differences in the mRNA expression of $B A X, B c l-2, B A X / B c l-2$ ratio and Fas were observed between the four groups of rats (Figure 7A and Figure

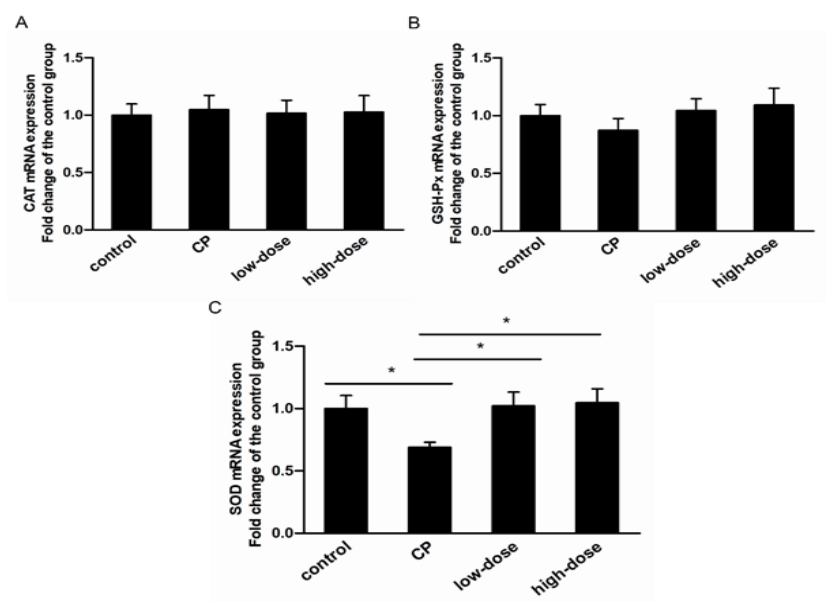

Figure 4: Ovarian mRNA expression of CAT, SOD and GSH-PX in rats. A) CAT; B) GSH-Px; C) SOD. Control, control group; $\mathrm{CP}$, cyclophosphamide (CP) group; low-dose, the low-dose SPPA and CP group; high-dose, the high-dose SPPA and CP group. Data was fold-change of the control group. Values are shown as mean \pm SEM and each group had 8 rats. * represent statistically significant differences $(P<0.05)$. 
7B). However, the mRNA expression levels of FasL and caspase- 3 were significantly up-regulated $(P<0.05)$ (Figure 7C) and ki67 level was down regulated $(P<0.05)$ (Figure 7D) in the CP group and these genes remained unaltered in the high- and low-dose groups as compared

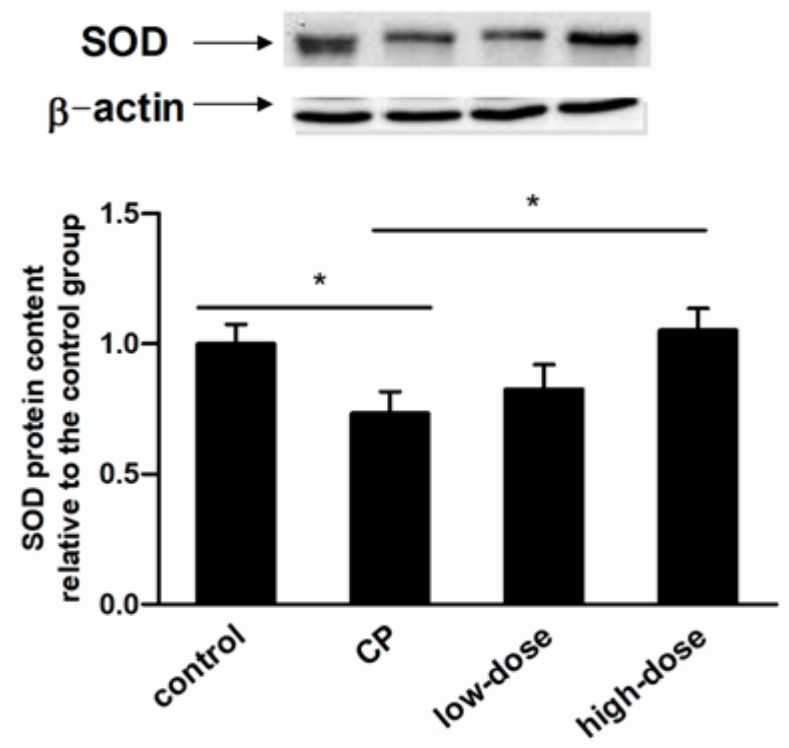

Figure 5: The SOD protein level in rat ovary. Control, the control group; CP, the cyclophosphamide (CP) group; lowdose, the low-dose SPPA and CP group; high-dose, the highdose SPPA and CP group. Data was fold change of the control group. Values are shown as mean \pm SEM and each group had 8 rats. ${ }^{*}$ represent statistically significant differences $(P<0.05)$.

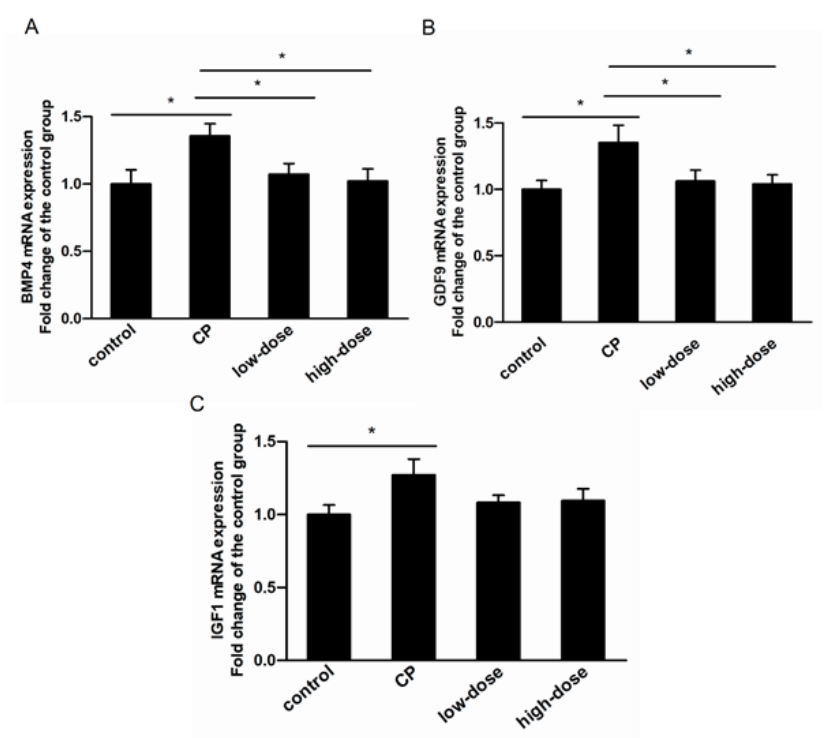

Figure 6: Ovarian expression of BMP4, GDF9 and IGF1 mRNA in rats. A) BMP4; B) GDF9; C) IGF1. Control, control group; $\mathrm{CP}$, the Cyclophosphamide (CP) group; low-dose, the lowdose SPPA and CP group; high-dose, the high-dose SPPA and CP group. Data was fold-change of the control group. Values are shown as mean \pm SEM and each group had 8 rats. * represent statistically significant differences $(P<0.05)$. to the control group of rats. Caspase-3 mRNA expression levels were significantly down regulated and ki67 mRNA expression levels were significantly increased $(P<0.05)$ in the high- and low-dose groups of rats as compared to the $\mathrm{CP}$ group of rats (Figure $7 \mathrm{C}$ and Figure 7D). In addition, Fasl expression was significantly down-regulated in the $(P<0.05)$ high-dose group of rats (Figure 7B).

\section{DISCUSSION}

The generation of reactive oxidative species resulting from imbalanced oxidant/antioxidant levels resulting in tissue destruction is termed oxidative stress. CP and its toxic metabolites hamper the intracellular antioxidant system, which culminates in ovarian oxidative stress. ${ }^{12}$ $\mathrm{CP}$ metabolites generate ROS in conjugation with GSH, which disrupts the antioxidant defense system resulting in ovarian dysfunction. ${ }^{14}$ Previous reports demonstrated an enhanced level of lipid peroxidation marker, MDA, in the ovary of rats treated with CP. ${ }^{17,18}$ Lipid peroxidation is the most detrimental effect of free radicals. ${ }^{19} \mathrm{CP}$, an alkylating agent, causes oxidative damage to the ovarian tissue and thus increases MDA and decreases GSH levels. ${ }^{20} \mathrm{CP}$ can also quickly deplete the oocyte reserves through cellular apoptosis, reducing the count of resting primordial and growing follicles in humans, resulting in early menopause, reduced reproductive functions, ${ }^{21}$ along with the altered quality of follicles. ${ }^{22}$

In the current study also, CP-treated rats exhibited reduced ovary weight, in line with the previous studies, ${ }^{12,23}$ along with reduced primordial, primary, secondary, antral and Graafian follicle, ovarian GSH-Px and SOD levels and increased ovarian MDA and NO levels. The cytotoxic metabolites of CP resulted in oxidative stress by raising the ROS levels leading to cellular lipid peroxidation, granulosa cell apoptosis and antral follicle atresia. ${ }^{1824}$ It has also been reported that increased levels of free radicals and reduced levels of antioxidants induce apoptosis of granulosa cells in antral follicular follicles. ${ }^{17}$ It indicated that CP could be used as a drug as per the oxidative stress models of the ovarian organ.

SOD converts $\mathrm{O}_{2}-$ to $\mathrm{H}_{2} \mathrm{O}_{2}$, which is subsequently converted to $\mathrm{H}_{2} \mathrm{O}$ by GSH-Px 10). GSH-Px alleviates the toxicity of oxidized GSH by catalyzing its conversion to reduced GSH. ${ }^{25}$ Besides, SOD is crucial for follicular development. The preovulatory follicles count was reduced in the ovaries of $\mathrm{SOD}_{1}$ null mice, which turned these mice subfertile. ${ }^{12,24}$ Also, copper chaperone-treated SOD null mice showed abnormal antral follicles in the ovary. ${ }^{24}$ In this study, CP treated rats showed significant down-regulation of SOD mRNA and SOD protein 
A

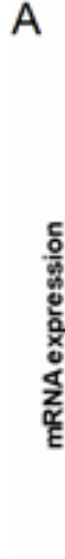

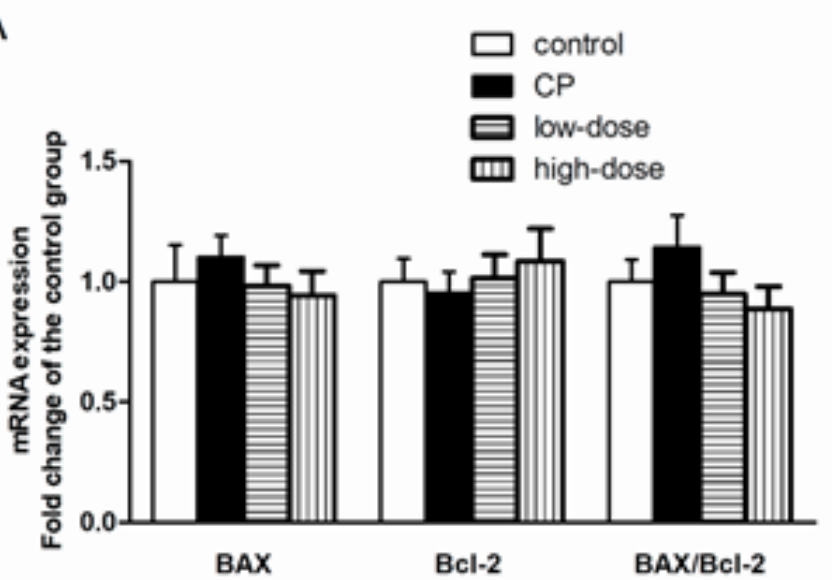

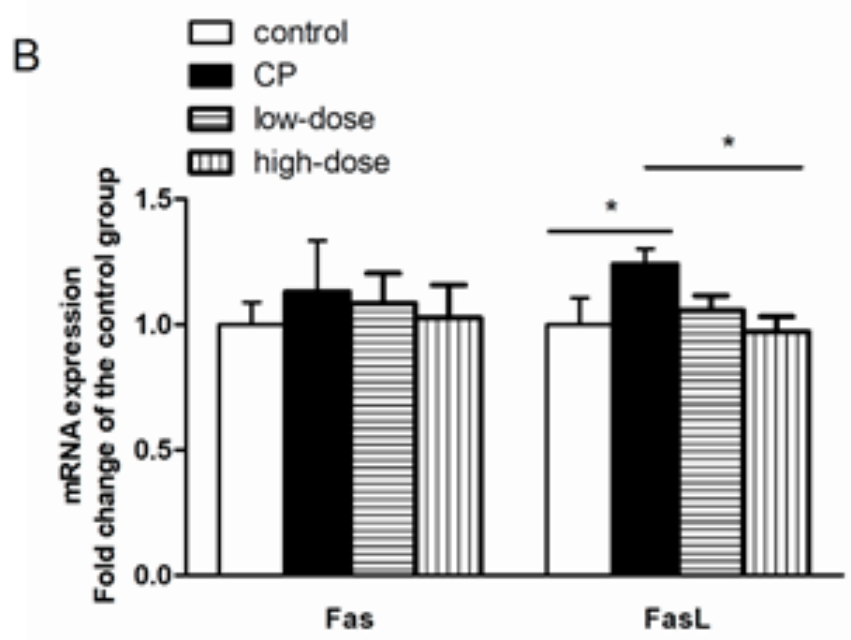

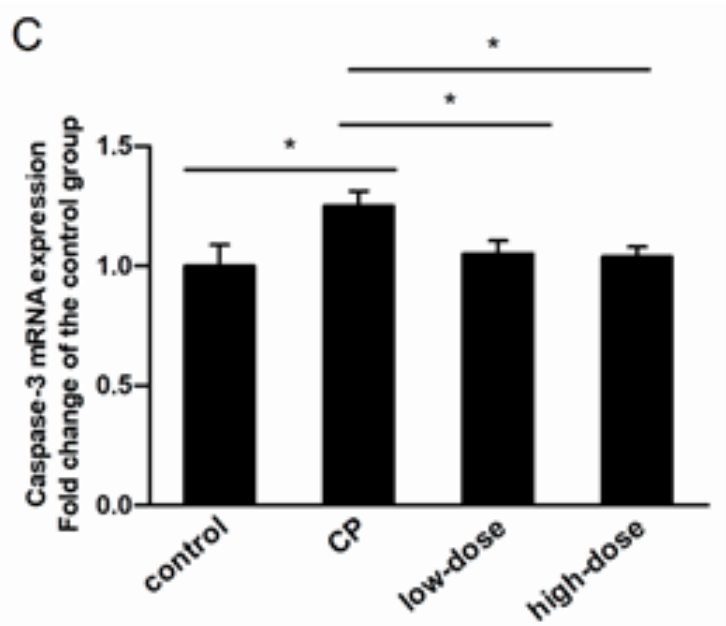

D

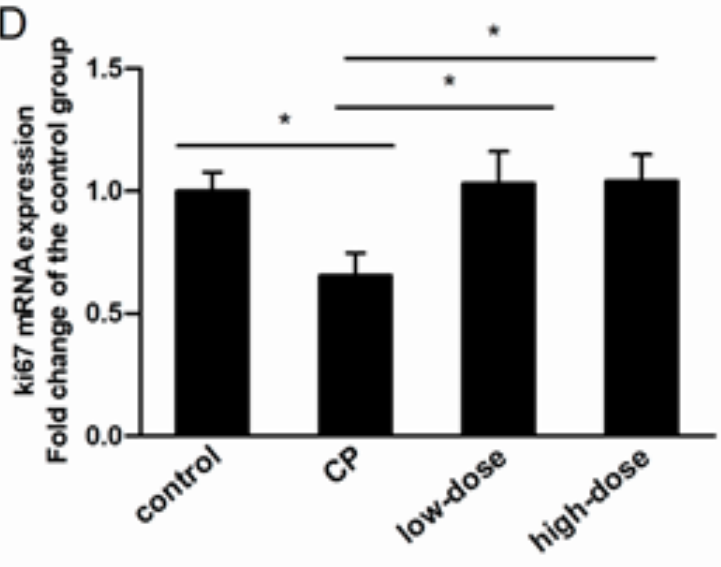

Figure 7: The mRNA expression of ovarian apoptosis and proliferation-related genes in rats. A) $B A X$, Bcl-2 and the ratio of $B A X /$ Bcl-2; B) Fas, FasL; C) Caspase-3; D) Ki67. Control, the control group; CP, the cyclophosphamide (CP) group; low-dose, the low-dose SPPA and CP group; high-dose, the high-dose SPPA and CP group. Data was fold-change of the control group. Values are shown as mean \pm SEM and each group had 8 rats. * represent statistically significant differences $(P<0.05)$.

levels as compared to the control rats. However, no significant differences in SOD mRNA and SOD protein levels were observed in the high- and low-dose groups of rats. Moreover, SOD mRNA and protein expression levels were significantly up-regulated in the high- and/or low-dose groups of rats as compared to the CP group of rats.

To the best of our knowledge, this is the first report to demonstrate antioxidative effects of SPPA in the CP-induced oxidative damage in the ovary of female rats. SPPA could be used to treat the SD female rats afflicted with oxidative damage since it can alter the expression levels of developmental stress-related genes. Besides, this study demonstrated the efficacy of SPPA on ovarian development and functions in rats. In this study, CP obliterated all the normal follicles; however, it increased the number of atretic follicles. It suggests that SPPA accelerated the development of primordial follicles from antral follicles; however, it arrested the follicle's maturation from antral to Graafian follicles and increased the atresia of Graafian follicles. In a previous study by Khedr $\mathrm{NF}^{12}$ rats treated with CP demonstrated similar changes in follicular development, which culminated in infertility. However, SPPA improved the adverse effects of CP-induced oxidative stress in ovaries and promoted follicular development and maturation. In the current study, SPPA was used as an antioxidative agent to counteract ovarian oxidative damage. It significantly ameliorated the histological and biochemical changes in rat ovaries and reduced NO and MDA levels. The number of Graafian follicles and ovarian weights in both SPPA groups increased compared to CP treated rats. It indicated the protective effect of SPPA on CP-induced oxidative damage in rat ovaries.

As per the previous reports, ${ }^{26}$ germline stem cells differentiate to form primordial follicles in the ovaries of adult animals. However, most of the researcher remains of the view that female animals contain primordial 
follicles since birth and each primordial follicle has three states: a) stationary state, b) activation followed by dormancy, c) development, maturation and ovulation.

Primordial follicles develop into Graafian follicles and the process involves its transformation to primary follicles, secondary follicles and antral follicles. To a certain extent, the number of Graafian follicles determines the reproductive efficiency of the females. In the current study, we observed that SPPA reduced the adverse effects of CP in both high- and lowdose groups of rats, reduced the atretic follicle count and increased the levels of Graafian follicles. These outcomes indicated that SPPA accelerated the follicular development in rats, improved the formation of mature follicles and increased the number of ovulatory follicles in rats. To validate these outcomes, the expression levels of genes related to ovarian development, apoptosis and proliferation were examined.

Ovarian follicle development primarily depends on the balance between cellular proliferation and apoptosis. GDF9 plays a crucial role in regulating the differentiation and growth of early oocytes. Previous studies in mice and sheep demonstrated that even if ovaries form primordial follicles and primary follicles, further development of follicles gets stagnated at the primary stage of monolayer granulosa cells due to GDF9 deficiency, which substantially mitigated the reproductive efficiency of the mother. ${ }^{27} \mathrm{BMP} 4$, a follicle survival factor, is involved in promoting the development of primordial follicles. ${ }^{28}$ Previous studies have demonstrated that the proportion of primary follicles in rat ovaries increased substantially and primordial follicles decreased significantly when cultured in vitro with exogenous BMP4. ${ }^{27}$ Conversely, the number of primary follicles decreased and primordial follicles increased significantly in mice treated with the BMP4 antibodies. ${ }^{29}$ In a study by Bachelot A et al. 2002, IGF-I knockout mice showed halted development and ovulation in the non-antral follicular phase or the early stage of antral follicles, indicating the involvement of IGF-I in folliculogenesis.

Follicular atresia is a normal physiological process in the ovary, where granulosa cells are eliminated through cellular apoptosis. ${ }^{30}$ SPPA treatment did not significantly affect mRNA expression levels of $B A X, B c l-2$ and $B A X / B c l-2$ ratio in the high- and low-dose groups of rats. However, post-SPPA treatment, the high-dose group showed significant upregulation of ovarian proliferation-related gene Ki67 and both the high- and low-dose group showed significant downregulation of ovarian apoptosis-related genes caspase-3 and Fasl. In this study, the mRNA expression levels of GDF9, $B M P 4$ and IGF-I were significantly up-regulated in the ovary of CP-treated rats and remained unaltered in the high- and low-dose groups of rats as compared to the control group of rats. These outcomes, along with the number of follicles, indicated that SPPA could accelerate the growth and maturation of ovarian follicles, but CP could accelerate follicle growth and terminates in antral follicles caused by cell apoptosis.

\section{CONCLUSION}

SPPA significantly mitigated the levels of MDA and $\mathrm{NO}$ in rat blood and thus enhanced the efficiency of the free radical scavenging and antioxidative system. SPPA also regulated the MDA levels generated by excessive free radicals. It prevented the MDA-induced damage to polyunsaturated fatty acids in the biological membrane and the cell membrane was protected from damage caused by excessive free radicals, ensuring tissue integrity. It indicated that SPPA ameliorated the adverse effects of NO, MDA and enhanced the SOD gene and SOD protein expression levels, which might be considered as the underlying mechanism for antioxidative effects of SPPA. SPPA could accelerate the growth of ovarian follicles in rats and promote the formation of mature follicles through DF9, BMP4 and IGF-I genes. The outcomes of the current study demonstrated that SPPA prevented CP-induced oxidative damage in rat ovaries. However, this is only a preliminary study on the follicles and gene expression related to ovarian development, oxidative stress, cellular apoptosis and proliferation; however, the associated molecular mechanism demands in-depth analysis.

\section{ACKNOWLEDGEMENT}

SPPA was provided by the Professor of Guangming Liu in Dali University, China (no. 20200830). This work was supported by a Grant from the National Natural Science Foundation of China (Grant NO.31760719). We are grateful to Xin Yu from the college of basic medicine at Dali University for his inputs. The authors would like to thank all the reviewers who participated in the review and MJEditor (www.mjeditor.com) for its linguistic assistance during the preparation of this manuscript.

\section{CONFLICT OF INTEREST}

No conflict of interest is associated with this work.

\section{ABBREVIATIONS}

P. americana: Periplaneta americana; SPPA: Small Peptides from P. americana; CP: cyclophosphamide; 
ROS: reactive oxygen species; NO: Nitric oxide; GSH-Px: glutathione peroxidase; CAT: Catalase; SOD: superoxide dismutase; MDA: malondialdehyde; GSSG: glutathione disulfide; NADPH: Nicotinamide adenine dinucleotide phosphate; GDF-9: Growth differentiation factor-9; Bcl-2: B-cell lymphoma-2; BMP4: Bone morphogenetic protein 4; IGF1: insulin-like growth factor 1; Da: Dalton; PH: Pouvoir Hydrogène; GAPDH: Glyceraldehyde-3-phosphate dehydrogenase; BW: body weight; OW: ovary weight; qRT-PCR: Reverse transcription-quantitative polymerase chain reaction; SEM: Scanning Electron Microscope; ${ }^{\circ} \mathrm{C}$ : Degree Centigrade; $\boldsymbol{\mu L}$ : Microliter; $\mu \mathrm{g}$ : Microgram.

\section{REFERENCES}

1. Lu J, Wang Z, Cao J, Chen Y, Dong Y. A novel and compact review on the role of oxidative stress in female reproduction. Reprod Biol Endocrinol. 2018;16(1):80.

2. Shaeib F, Khan SN, Ali I, Thakur M, Saed MG, Dai J, et al. The Defensive Role of Cumulus Cells against Reactive Oxygen Species Insult in Metaphase II Mouse Oocytes. Reprod Sci. 2016;23(4):498-507.

3. Agarwal A, Saleh RA, Bedaiwy MA. Role of reactive oxygen species in the pathophysiology of human reproduction. Fertil Steril. 2003;79(4):829-43.

4. Zeng C, Liao Q, Hu Y, Shen Y, Geng F, Chen L. The Role of Periplaneta americana (Blattodea: Blattidae) in Modern Versus Traditional Chinese Medicine. J Med Entomol. 2019;56(6):1522-6.

5. Yu S, Zhang H, Zhang T, Liu J. Research advances in pharmacological action and clinical application of Periplaneta americana. Liaoning Coll Tradit Chin Med. 2016;13(4):228-30.

6. Gao Y, Fang X, Vincent DF, Threadgill DW, Bartholin L, Li Q. Disruption of postnatal folliculogenesis and development of ovarian tumor in a mouse model with aberrant transforming growth factor beta signaling. Reprod Biol Endocrinol. 2017;15(1):94.

7. Zhang L, Wang H, Yu D, Chen J, Xing C, Li J, et al. The effects of mouse ovarian granulosa cell function and related gene expression by suppressing BMP/Smad signaling pathway. Anim Cells Syst. 2018;22(5):317-23.

8. Monte APO, Barros VRP, Santos JM, Menezes VG, Cavalcante AYP, et al. Immunohistochemical localization of insulin-like growth factor-1 (IGF-1) in the sheep ovary and the synergistic effect of IGF-1 and FSH on follicular development in vitro and $\mathrm{LH}$ receptor immunostaining. Theriogenology. 2019;129:61-9.

9. Rucker EB $3^{\text {rd }}$, Dierisseau P, Wagner KU, Garrett L, Wynshaw-Boris A, Flaws $\mathrm{JA}$, et al. Bcl-x and Bax regulate mouse primordial germ cell survival and apoptosis during embryogenesis. Mol Endocrinol. 2000;14(7):1038-52.

10. Liu J, Jiang Y, Mao J, Gu B, Liu H, Fang B. High levels of glucose induces a dose-dependent apoptosis in human periodontal ligament fibroblasts by activating caspase-3 signaling pathway. Appl Biochem Biotechnol. 2013;170(6):1458-71.

11. Dolmans MM, Yuan WY, Camboni A, Torre A, Langendonckt VA, MartinezMadrid B, et al. Development of antral follicles after xenografting of isolated small human preantral follicles. Reprod Biomed Online. 2008;16(5):705-11.
12. Khedr NF. Protective effect of mirtazapine and hesperidin on cyclophosphamide-induced oxidative damage and infertility in rat ovaries. Exp Biol Med. 2015;240(12):1682-9

13. Mark-Kappeler CJ, Hoyer PB, Devine PJ. Xenobiotic effects on ovarian preantral follicles. Biol Reprod. 2011;85(5):871-83.

14. Tsai-Turton M, Luong BT, Tan Y, Luderer U. Cyclophosphamide-induced apoptosis in COV434 human granulosa cells involves oxidative stress and glutathione depletion. Toxicol Sci. 2007;98(1):216-30.

15. Cheng G, Weihua Z, Mäkinen S, Mäkelä S, Saji S, Warner M, et al. A role for the androgen receptor in follicular atresia of estrogen receptor beta knockout mouse ovary. Biol Reprod. 2002;66(1):77-84.

16. Li LA, Xia D, Wei S, Hartung J, Zhao RQ. Characterization of adrenal ACTH signaling pathway and steroidogenic enzymes in Erhualian and Pietrain pigs with different plasma cortisol levels. Steroids. 2008;73(8):806-14.

17. Yener NA, Sinanoglu O, Ilter E, Celik A, Sezgin G, Midi A, et al. Effects of spirulina on cyclophosphamide-induced ovarian toxicity in rats: Biochemical and histomorphometric evaluation of the ovary. Biochem Res Int. 2013;2013:764262.

18. Saleh DO, Mansour DF. Ovario-protective effects of genistein against cyclophosphamide toxicity in rats: Role of anti-müllerian hormone and oestradiol. Eur J Pharmacol. 2016;789:163-71.

19. Girotti AW. Lipid hydroperoxide generation, turnover and effector action in biological systems. J Lipid Res. 1998;39(8):1529-42.

20. Borekci B, Yapca OE, Turan MI, Gul MA, Isaoglu U. Prevention of ovarian oxidative stress-related infertility associated with cisplatin in rats with thiamine pyrophosphate. Lat Am J Pharm. 2013;32:512-7.

21. Lemos CN, Reis FM, Pena GN, Silveira LC, Camargos AF. Assessment of fertility protection and ovarian reserve with $\mathrm{GnRH}$ antagonist in rats undergoing chemotherapy with cyclophosphamide. Reprod Biol Endocrinol. 2010;8(1):51.

22. Yuksel A, Bildik G, Senbabaoglu F, Akin N, Arvas M, Unal F, et al. The magnitude of gonadotoxicity of chemotherapy drugs on ovarian follicles and granulosa cells varies depending upon the category of the drugs and the type of granulosa cells. Hum Reprod. 2015;30(12):2926-35.

23. Plowchalk DR, Meadows MJ, Mattison DR. Reproductive toxicity of cyclophosphamide in the C57BL/6N mouse: 2. Effects on uterine structure and function. Reprod Toxicol. 1992;6(5):423-9.

24. Devine PJ, Perreault SD, Luderer U. Roles of reactive oxygen species and antioxidants in ovarian toxicity. Biol Reprod. 2012;86(2):27.

25. Wu J, Zhao F, Wang M, Xi B. The Research of Variety of SOD GSH CAT and MDA in Cyclophosphamide Injuring Mice's Serum Resulted from the Effect of Sijunzi Decoction. Liaoning J Tra Chi Med. 2007;34:20.

26. Johnson J, Bagley J, Skaznik-Wikiel M, Lee HJ, Adams GB, Niikura Y, et al. Oocyte generation in adult mammalian ovaries by putative germ cells in bone marrow and peripheral blood. Cell. 2005;122(2):303-15.

27. Bachelot A, Monget P, Imbert-Bolloré P, Coshigano K, Kopchick JJ, Kelly PA, et al. Growth hormone is required for ovarian follicular growth. Endocrinology. 2002;143(10):4104-12.

28. Nilsson EE, Skinner MK. Bone morphogenetic protein-4 acts as an ovarian follicle survival factor and promotes primordial follicle development. Biol Reprod. 2003;69(4):1265-72.

29. Tanwar PS, O'Shea T, McFarlane JR. In vivo evidence of role of bone morphogenetic protein-4 in the mouse ovary. Anim Reprod Sci. 2008;106(34):232-40.

30. Ding W, Wang W, Zhou B, Zhang W, Huang P, Shi F, et al. Formation of primordial follicles and immunolocalization of PTEN, PKB and FOXO3A proteins in the ovaries of fetal and neonatal pigs. J Reprod Dev. 2010;56:1628. 
PICTORIAL ABSTRACT

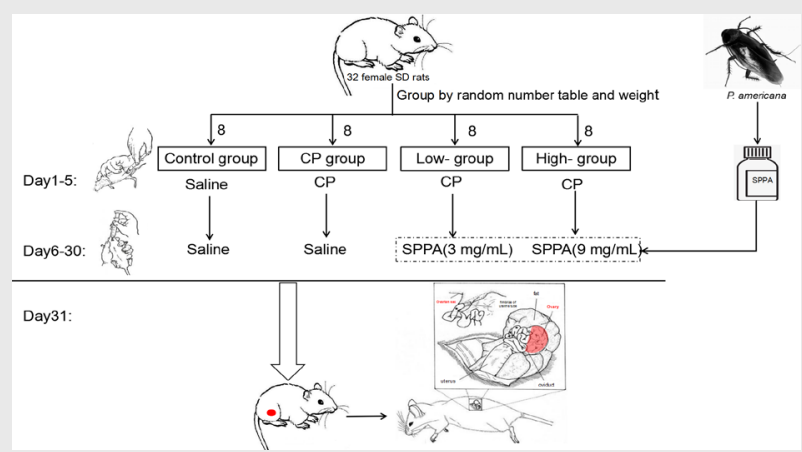

\section{SUMMARY}

The purpose of this study is to evaluate the effects of Small Peptides from P. americana (SPPA) on ovarian follicles and antioxidant capacity in rats. In vivo model of oxidative stress, i.e., Sprague-Dawley (SD) rats with cyclophosphamide (CP)-induced oxidative stress, was used to evaluate the effects of SPPA. The fresh product of Periplaneta americana was extracted by alcohol-water extraction, concentration, degreasing, macroporous adsorption resin column chromatography, alcohol solvent elution and other procedures to obtain a peptide-based refined extract (ie SPPA), which was identified Small peptides in this substance account for $94.44 \%$. The outcomes of this study will extend our understanding of the antioxidative effect of SPPA in rat ovaries. Also, it will provide platform data to reveal the impact of SPPA on reproductive functions.

\section{About Authors}

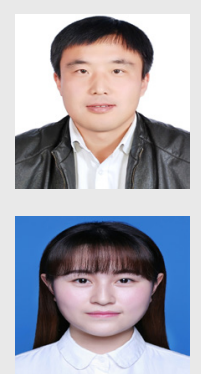

Dr. Shiyan Sui, Associate Professor of School of Public Health, Dali University, Yunnan, China. $\mathrm{He}$ is having 13 yrs of teaching experience. Presently he is a Research Director in Experimental Teaching Center and Health Research Center, School of Public Health, Dali University. He is a Ph.D. engaged in researching vegetative reproduction mechanisms and pesticide action mechanisms.

Qin Wang, studying Postgraduate in Dali University, Yunnan, China, carrying out the research work under the guidance of Prof. Shiyan Sui. She has been accepted 2 review articles in reputed national Journals, and has been accepted 2 research articles in Science Citation Index (SCI).

Cite this article: Wang Q, Kong C, Liu K, Fu R, Si H, Sui S. Protective Effects of Small Peptides from Periplaneta americana on Cyclophosphamide-induced Oxidative Stress in Rat Ovaries. Indian J of Pharmaceutical Education and Research. 2021;55(3):782-92. 